\title{
miRNA-181b increases the sensitivity of pancreatic ductal adenocarcinoma cells to gemcitabine in vitro and in nude mice by targeting BCL-2
}

\author{
BAOBAO CAI ${ }^{1}$, YONG AN $^{1}$, NAN LV $^{1}$, JIANMIN CHEN $^{2}$, MIN TU $^{1}$, JIE SUN $^{1}$, \\ PENGFEI WU ${ }^{1}$, JISHU WEI ${ }^{2}$, KUIRONG JIANG ${ }^{2}$ and YI MIAO ${ }^{2}$ \\ ${ }^{1}$ Institute of Tumor Biology, Jiangsu Province Academy of Clinical Medicine; ${ }^{2}$ Department of General Surgery, \\ The First Affiliated Hospital of Nanjing Medical University, Nanjing, Jiangsu 210029, P.R. China
}

Received October 21, 2012; Accepted December 27, 2012

DOI: 10.3892/or.2013.2297

\begin{abstract}
Pancreatic ductal adenocarcinoma (PDAC) is a highly lethal disease and is usually resistant to chemotherapy. MicroRNA-181b (miR-181b) has been reported to be associated with chemoresistance in various types of cancer. In this study, we investigated the effects of miR-181b on the chemosensitivity of PDAC cells to gemcitabine and the underlying molecular events. miR-181b mimics and inhibitors were synthesized for transient gene transfection in vitro. Lentivirus carrying miR-181b mimics were used to infect PDAC cells for nude mouse xenograft assays by implanting infected PDAC cells into recipient mice. Cell viability was determined by MTT assays, while gene expression was assessed using qRT-PCR, western blot analysis and enzymelinked immunosorbent assay (ELISA). miR-181b targeting BCL-2 expression was assessed by a dual-luciferase activity assay. The data showed that miRNA-181b expression sensitized PDAC cells to gemcitabine treatment. Although gemcitabineresistant PDAC cell sublines (SW1990/GR and CFPAC-1/GR) expressed higher levels of miRNA-181b, gemcitabine induced higher levels of apoptosis in PDAC cells transfected with miRNA-181b mimics. The nude mouse xenograft assay data showed that miR-181b transfection also sensitized the cells to gemcitabine treatment in vivo. Molecularly, bioinformatics data predicted that miR-181b was able to bind to BCL-2 mRNA 3'UTR. The dual luciferase activity assay revealed that miRNA-181b downregulated BCL-2 expression. The results from western blot analysis showed a reduced BCL-2 expression following miR-181b transfection but an enhanced caspase-3 activity in miRNA-181b mimic-transfected PDAC cells. This
\end{abstract}

Correspondence to: Dr Kuirong Jiang or Dr Yi Miao, Department of General Surgery, The First Affiliated Hospital of Nanjing Medical University, 300 Guangzhou Road, Nanjing, Jiangsu 210029, P.R. China E-mail: jiangkuirong@njmu.edu.cn

E-mail: miaoyi@njmu.edu.cn

Key words: pancreatic ductal adenocarcinoma, microRNA-181b, gemcitabine resistance, BCL-2 study demonstrates that miRNA-181b sensitizes PDAC cells to gemcitabine by targeting BCL-2.

\section{Introduction}

Pancreatic ductal adenocarcinoma (PDAC) is the most lethal human malignancy worldwide and has a very low 5-year-survival rate (approximately 6\%). In the US alone, approximately 40,000 patients succumbed to the disease in 2011 (1). The majority of PDAC patients are diagnosed at advanced stages, since the disease does not cause specific symptoms during the early stages. To date, surgery is the primary treatment for pancreatic cancer. By contrast, chemotherapy is only used for patients who are not suitable for surgical resection with curative intent. A frequently used chemotherapeutic drug is gemcitabine, which can effectively improve quality of life and increase patient survival. Nevertheless, the high rate of resistance to gemcitabine contributes to the poor prognosis of pancreatic cancer $(2,3)$. Thus, the development of novel therapeutic strategies is mandatory.

The development of pancreatic cancer, like most other cancer types of cancer, involves the activation of oncogenes and the inactivation of tumor suppressor genes, dysregulating signaling proteins critical to cell growth. Thus, the effective control of pancreatic cancer cell growth or the induction of apoptosis should target multiple gene pathways. Otherwise, drug resistance will eventually develop. The molecular mechanisms responsible for the resistance of PDAC patients to gemcitabine may be due to: i) An altered tumor microenvironment, such as a dense desmoplastic stroma, preventing gemcitabine infusion to the tumor parenchyma. A previous study demonstrated that tumors from gemcitabine-resistant patients had increased stimulation of stroma-related gene pathways (4). ii) The key molecular pathway responsible for gemcitabine metabolism is altered; thus, the activation of gemcitabine is anomalously inhibited or the excretion of gemcitabine is accelerated. A previous study demonstrated that the inactivation and downregulation of deoxycytidine kinase, a key enzyme in gemcitabine activation, plays a significant role in acquiring gemcitabine resistance (5). Moreover, transmembrane xenobiotic transporters, such as ATP-binding cassette sub-family G member 2 (ABCG-2), 
play a role in gemcitabine resistance (6). iii) The activation of aberrant proliferative and apoptotic pathways may occur; thus, drug-resistant PDAC cells have a survival advantage against chemotherapy $(7,8)$.

In order to overcome drug resistance, research is focusing on microRNAs (miRNAs or miRs). miRNAs are a family of naturally occurring, non-coding RNA molecules, 19 to 25 nucleotides in length. miRNAs post-transcriptionally regulate the expression of target genes $(9,10)$. Thus, miRNAs participate in a wide range of biological processes, such as embryonic development, organ formation and cell proliferation and apoptosis (11). miRNAs also play an important role in tumorigenesis and chemosensitivity (12-14). miR-181b has been found to be downregulated in glioblastoma and has been widely studied in a variety of human cancers. For example, in urothelial carcinoma (15), thyroid papillary carcinoma (16), acute lymphocytic leukemia (17), chronic lymphocytic leukemia (CLL) (18), colorectal cancer (19), breast cancer (20), prostate cancer (21), retinoblastoma (22) and PDAC (23), miRNA-181b is upregulated. Moreover, its expression is reduced in glioblastoma (24) and gastric cancer (25). The loss of miRNA-181b expression may contribute to the resistance of leukemia cells to chemotherapy (18). miRNA-181b is expressed during the stable stages of CLL; however, a reduction in miRNA-181b expression has been associated with disease progression and drug refraction (18).

Other studies have shown that miRNA-181b significantly enhances drug sensitivity and its underexpression has been associated with a shorter treatment-free survival in CLL cells (26). However, another study reported the downregulation of miRNA-181b in chemotherapy-responsive acute promyelocytic leukemia (27). miRNA-181b expression is increased in chemoresistant hepatocellular carcinoma (28), colorectal cancer (29) and breast cancer (30), as well as in drug-refractory gastric and lung cancers (31). By targeting different genes, miRNA-181b may play a complicated role in chemotherapyresistance, depending on the tumor type and anti-neoplastic agent (12). In this study, we investigated whether miR-181b is associated with the sensitivity of PDAC cells to gemcitabine in vitro and in nude mouse xenografts.

\section{Materials and methods}

Cell lines and culture. The PDAC SW1990 and CFPAC-1 cell lines were obtained from Shanghai Cell Bank (Shanghai, China). These cell lines were propagated and cultured in Dulbecco's modified Eagle's medium (DMEM, Invitrogen, Carlsbad, CA, USA), supplemented with $10 \%$ fetal bovine serum (FBS; Sigma, St. Louis, MO, USA), $100 \mu \mathrm{g} / \mathrm{ml}$ penicillin and streptomycin, in a humidified chamber at $37^{\circ} \mathrm{C}$ with $5 \% \mathrm{CO}_{2}$.

Gemcitabine-resistant PDAC SW1990/GR and CFPAC-1/GR cell sublines were acquired by culturing the parental cells with gradually increasing concentrations of gemcitabine (Lilly, Neuilly-sur-Seine Cedex, France) for approximately 6 months as documented in a previous study (32). In brief, the cells were cultured in gemcitabine-conditioned medium for 3 days at the concentration of $3 \mu \mathrm{M}$, followed by a recovery step, and agentfree medium culturing until the cells recovered exponential growth. MTT assays were performed to evaluate the $\mathrm{IC}_{50}$ of the gemcitabine-treated cells. The $\mathrm{IC}_{50}$ dose was used for the gemcitabine-conditioned medium to treat the cells. By increasing the dosage of gemcitabine in the culture medium intermittently for approximately 6 months (24 weeks for SW1990 and 21 weeks for CFPAC-1 cells), stable gemcitabine-resistant cell sublines (SW1990/GR and CFPAC-1/GR) were acquired. The $\mathrm{IC}_{50}$ in the SW1990/GR and CFPAC-1/GR cells was 232.2 and $314.4 \mu \mathrm{M}$, respectively.

miRNA mimics and inhibitor, gene transfection, miRNAoverexpressed lentivirus and virus infection. miR-181b mimics, inhibitor and negative control were designed and synthesized by GenePharma Co., Ltd. (Shanghai, China). The primer sequences are shown in Table I. For gene transfection, the cells were cultured in 6-well plates to $40 \%$ confluence. miR-181b mimics, inhibitor and negative control were mixed with Lipofectamine 2000 (Invitrogen), and then added to the cell culture medium according to the manufacturer's instructions. After $24 \mathrm{~h}$ of transfection, total RNA and protein were prepared from the cells and subjected to qRT-PCR and western blot analyses, respectively.

A miRNA-181b lentivirus overexpression system was obtained from GenePharma. Briefly, the miRNA-181b lentiviral expression vectors, LV3-pGLV-H1-miRNA-181b-GFP-Puro and LV3-pGLV-H1-Null-GFP-Puro (null control) were constructed. The vectors were then transfected into 293-T cells. The supernatant titer was determined to be $1 \times 10^{8} \mathrm{TU} / \mathrm{ml}$. Subsequently, the lentivirus was used to infect PDAC cells following the manufacturer's instructions.

$q R T-P C R$. Total cellular RNA from the cultured cells was isolated using TRIzol reagent (Invitrogen). RNA samples (500 ng each) were then reverse-transcribed into cDNA with miRNA-181b reverse transcriptase primers (Applied Biosystems, Foster City, CA, USA) using a TaqMan MicroRNA Reverse Transcription kit (Applied Biosystems). Levels of miRNA-181b and U6 expression were determined by qPCR with TaqMan MicroRNA Assays (Applied Biosystems) and an ABI 7500 machine (Applied Biosystems). The levels of mature miRNA-181b expression were then normalized to U6 and calculated as the inverse log of the $\Delta \Delta \mathrm{CT}$. All procedures were performed following the manufacturer's instructions.

Protein extraction and western blot analysis. Total cell protein lysates were prepared using an RIPA buffer supplemented with $1 \%$ phenylmethylsulfonyl fluoride (PMSF). Protein concentration was determined by a BCA kit (Keygen, Nanjing, China). Subsequently, $30 \mu \mathrm{g}$ of protein sample each was separated by SDS-PAGE (10\% gels) and transferred onto a $0.45 \mu \mathrm{m}$ polyvinylidene fluoride (PVDF) membrane (Millipore, Bedford, USA) using a mini trans-blot system (Bio-Rad Laboratories, Hercules, CA, USA). Primary antibodies and corresponding secondary antibodies were then added followed by incubation. A rabbit anti-human BCL-2 antibody was purchased from Cell Signaling Technology (Danvers, MA, USA), and a mouse antihuman GAPDH antibody and goat anti-rabbit or anti-mouse secondary antibody were obtained from Beyotime (Nantong, China). To quantify the protein expression, protein expression was normalized to GAPDH levels (Chemilmager 5500; Alpha Innotech Corp., San Leandro, CA, USA). 
Table I. Sequences of miR-181b mimics, inhibitor and negative control.

Mimic (5'-3')

Inhibitor $\left(5^{\prime}-3^{\prime}\right)$

miRNA-181b

Negative control
AACAUUCAUUGCUGUCGGUGGGU UUCUCCGAACGUGUCACGUTT
ACCCACCGACAGCAAUGAAUGUU

Table II. Target sites of BCL-2 3'-UTR and their mutants or mismatch sequences.

\begin{tabular}{|c|c|}
\hline & Sequence $\left(5^{\prime}-3^{\prime}\right)$ \\
\hline WT2896 & CTTATTGTTAAAAACATGTTAGAAGCAATGAATGTATATAAAAGCCTCAACTAGTCATTT \\
\hline MT2896 & СTTATTGTTAAAAACATGTTAGAAGCAATTCCTACATATAAAAGCCTCAACTAGTCATTT \\
\hline WT1752 & ATACCATTTATCTGTATTAACTTTGGAATGTACTCTGTTCAATGTTTAATGCTGTGGTTG \\
\hline MT1752 & ATACCATTTATCTGTATTAACTTTGGCAGAGACTCTGTTCAATGTTTAATGCTGTGGTTG \\
\hline
\end{tabular}

MT, mutant; WT, wild-type. The full line indicates the wild-type putative binding sequences and the dotted line indicates the mutant sites of these sequences.

Enzyme-linked immunosorbent assay (ELISA). To detect caspase-3 activity, we first seeded the cells at a density of $1.5 \times 10^{6}$ cells per well in 6 -well plates. The cells were then transfected with miRNA-181b mimics, inhibitor or negative controls for $48 \mathrm{~h}$. Subsequently, caspase-3 activity was analyzed using a CaspACE Assay System (Promega, Madison, Wisconsin, USA) following the manufacturer's instructions.

Cell viability MTT assay. To detect cell viability, we first seeded $2 \times 10^{3}$ cells in 96-well plates, and then transfected them with miR-181b mimics, inhibitor or negative controls for $72 \mathrm{~h}$. Subsequently, $20 \mu 1$ per well of 3-(4,5-dimethythiazol-2-yl)2,5-diphenyl tetrazolium bromide (MTT) solution $(5 \mathrm{mg} / \mathrm{ml}$, Sigma) was added to the cells followed by incubation at $37^{\circ} \mathrm{C}$ for $4 \mathrm{~h}$. Then, $150 \mu \mathrm{l}$ of dimethyl-sulphoxide (DMSO) was substituted for the supernatant, followed by oscillation for $10 \mathrm{~min}$. Absorbance at $490 \mathrm{~nm}$ was detected using a microplate reader (Multiskan MK3; Thermo Labsystems, Franklin, MA, USA). All the results were normalized to the corresponding controls and the percentage of the control was calculated.

Apoptosis Annexin V/flow cytometric assay. Cells were incubated with culture medium containing gemcitabine at a final concentration of $0.1 \mu \mathrm{M}$ for $48 \mathrm{~h}$. Cell pellets were then collected, washed with phosphate-buffered saline (PBS), resuspended in $100 \mu \mathrm{l}$ of $1 \mathrm{X}$ binding buffer and stained with $5 \mu 1$ phycoerythrin-Annexin $\mathrm{V}$ and $5 \mu 1$ of 7-AAD (BectonDickinson, Franklin Lakes, NJ, USA) at room temperature for $15 \mathrm{~min}$ in the dark. A flow cytometer (Becton-Dickinson) was utilized to evaluate the apoptotic levels in each sample.

Dual-luciferase reporter assay. Luciferase reporter constructs carrying 60-bp-long synthetic oligonucleotides (Invitrogen, Shanghai, China) and containing wild-type putative miRNA binding sites from the human BCL-2 3'-UTR or their mutant versions (www.targetscan.org; Table II) were inserted in XbaI-
FseI sites of pGL3-control vectors (Promega). The construct was confirmed by DNA sequencing. Aliquots of $1.5 \times 10^{5}$ cells were seeded into 24-well plates. After 24 h, $200 \mathrm{ng}$ of each independent luciferase reporter plasmid plus $80 \mathrm{ng}$ of pRL-TK (Promega) plasmid as the control were co-transfected with $60 \mathrm{pmol}$ of the miRNA-181b mimics, inhibitor or control. Luciferase activity was then measured $48 \mathrm{~h}$ after transfection using the Dual-Luciferase Reporter Assay System with a GloMax Luminometer (Promega). Firefly luciferase activity was normalized to Renilla luciferase activity for each transfected cell sample.

In vivo chemosensitivity assay. For in vivo chemosensitivity analyses, 2x10 cells from the SW1990 and SW1990/GR cell lines transfected with LV3-pGLV-H1-miRNA-181bGFP-Puro, LV3-pGLV-H1-Null-GFP-Puro and mock control vectors were resuspended in $25 \mu 1$ of DMEM, and then injected into the pancreatic undercapsule in 4-week-old $\mathrm{BALB} / \mathrm{c}$ female nude mice (6 mice in each group). BALB/c female nude mice were purchased from The Model Animal Research Center of Nanjing University, Nanjing, China. Our animal studies were approved by the Ethics Committee of Nanjing Medical University. Two weeks after cell injection, gemcitabine $(150 \mathrm{mg} / \mathrm{kg})$ was injected intraperitoneally twice weekly for 28 days. At the end of the experiments, the mice were euthanized and the tumor lesions were excised. Tumor volume was determined as $\mathrm{V}=\left(\mathrm{Lx} \mathrm{W}^{2}\right) / 2$, where $\mathrm{L}$ represents the length of the tumor and $\mathrm{W}$ represents the width of the tumor.

Statistical analysis. All in vitro experiments were performed in triplicate and repeated at least once. The Student's t-test was performed to assess statistical differences between 2 groups and the F-test was used for comparisons among 3 or more groups. The $\mathrm{Q}$ test was used for multiple comparisons. A P-value $<0.05$ was considered to indicate a statistically significant difference. 
A

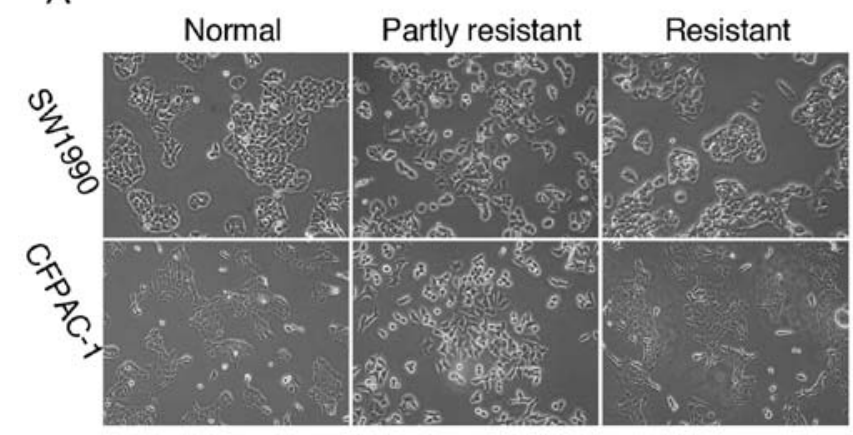

B

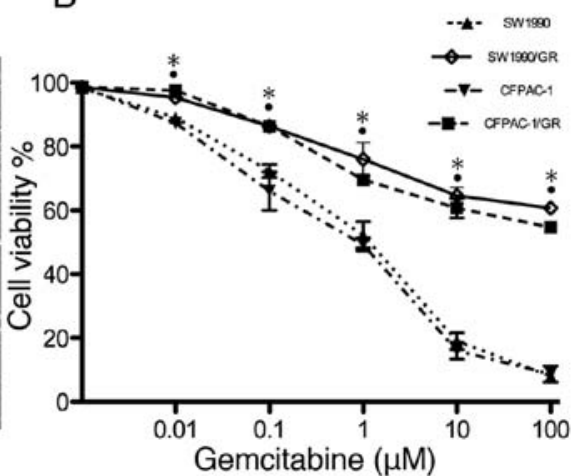

Figure 1. Establishment of gemcitabine-resistant pancreatic ductal adenocarcinoma (PDAC) SW1990 and CFPAC-1 sublines. (A) Morphology of parental and gemcitabine-resistant PDAC SW1990 and CFPAC-1 sublines. Compared to their parental cell lines, SW1990/GR and CFPAC-1/GR cells maintained a fibroblast-like phenotype with loosening intercellular contact and invadopodia formation (original magnification, x200). However, at the full resistance stage, the SW1990/GR and CFPAC-1/GR cell lines regained their parental morphology. (B) MTT assay. The graph shows the significantly enhanced gemcitabine resistance of SW1990/GR and CFPAC-1/GR cells at a drug concentration $>0.01 \mu \mathrm{M}$ ('SW1990 vs. SW1990/GR, p<0.05; $\bullet$, CFPAC-1 vs. CFPAC-1/GR, p<0.05).

A

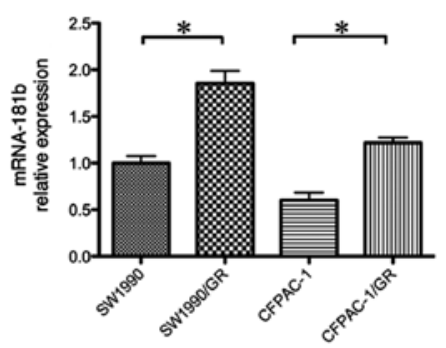

B

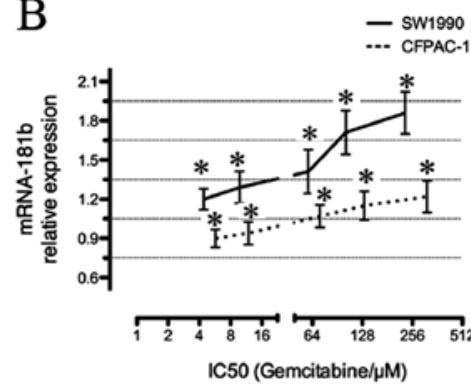

$\mathrm{C}$

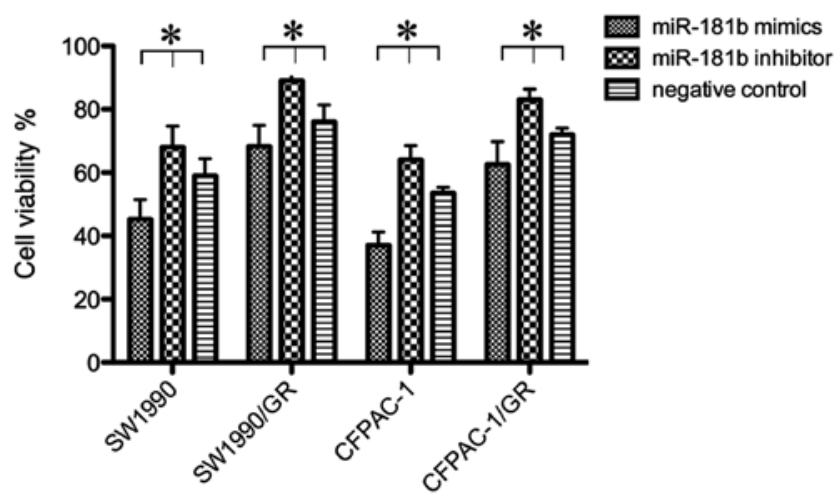

Figure 2. (A) qRT-PCR. Levels of miRNA-181b expression in PDAC cell lines (SW1990 vs. SW1990/GR: 1.00 vs. 1.86; CFPAC vs. CFPAC-1: 0.60 vs. 1.22; "p<0.05). (B) qRT-PCR. Expression of miRNA-181b was elevated with rising IC50 levels of gemcitabine ("p<0.05). (C) MTT assay. Cells were grown and transfected with miR-181b constructs, treated with gemcitabine, and then subjected to MTT assay. Cell viability was restrained in the cell lines transfected with miRNA-181b mimics. More cells survived in the gemcitabine milieu $(1 \mu \mathrm{M})$ when miRNA-181b was inhibited ( $\left.{ }^{*} \mathrm{p}<0.05\right)$.

\section{Results}

Establishment of gemcitabine-resistant pancreatic cancer cell sublines. In this study, we first established gemcitabineresistant pancreatic cancer cell sublines by cultivating SW1990 and CFPAC-1 cells with gradually increasing concentrations of gemcitabine for 6 months. At first, SW1990 and CFPAC-1 cells had partially increased viability with morphological changes, followed by permanent gemcitabine resistance with phenotypic recovery (Fig. 1A). The cell viability MTT assay confirmed the increased resistance (Fig. 1B).
miRNA-181b regulates gemcitabine resistance in PDAC cells. To assess the role of miR-181b in PDAC cells, we first determined the miRNA-181b expression in normal and resistant cell lines. miR-181b indeed was differentially expressed between the parental and resistant cell lines, suggesting that miR-181b affects gemcitabine sensitivity in PDAC cells. In particular, miRNA-181b was expressed in the SW1990 and CFPAC-1 cells, as detected by qRT-PCR (Fig. 2A). However, the SW1990/GR and CFPAC-1/GR cells expressed significantly higher levels of miRNA-181b (Fig. 2B). SW1990, CFPAC-1, SW1990/GR and CFPAC-1/GR cells were transiently trans- 
Table III. Effects of miR-181b on PDAC cell viability following treatment with gemcitabine $(1 \mu \mathrm{M})$ for $72 \mathrm{~h}(\%$ of control).

\begin{tabular}{lrrrr}
\hline & \multicolumn{4}{c}{ Cell line } \\
\cline { 2 - 5 } & SW1990 & SW1990/GR & CFPAC-1 & CFPAC-1/GR \\
\hline miRNA-181b mimics & $76.41 \%$ & $89.64 \%$ & $69.34 \%$ & $86.43 \%$ \\
miRNA-181b inhibitor & $114.15 \%$ & $117.71 \%$ & $120.94 \%$ & $116.62 \%$ \\
\hline
\end{tabular}

A
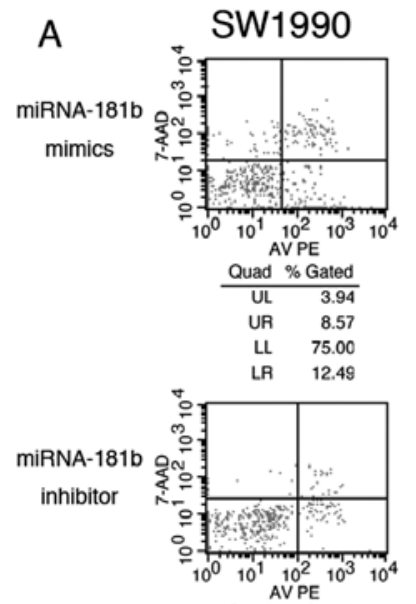

$\frac{\text { Quad \% Gated }}{\text { UL } 0.98}$

UR $\quad 2.71$

L $\quad \begin{aligned} 2.23 \\ \text { L }\end{aligned}$

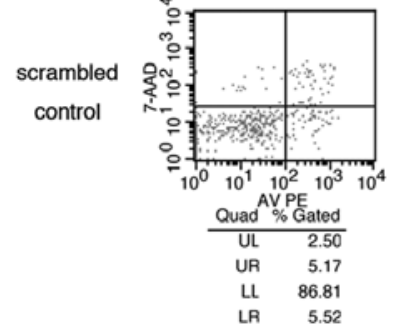

SW1990/GR
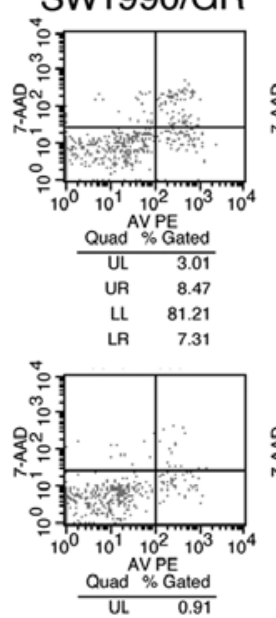

UR $\quad 1.89$

LL 92.84

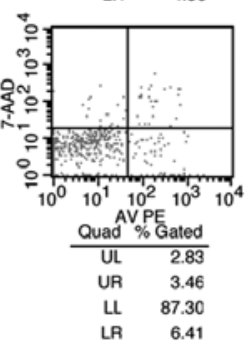

CFPAC-1

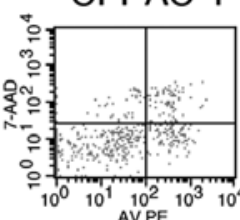

$\frac{\text { Quad } \% \text { PE Gated }}{4.75}$

UR 11.95

LL $\quad 71.41$
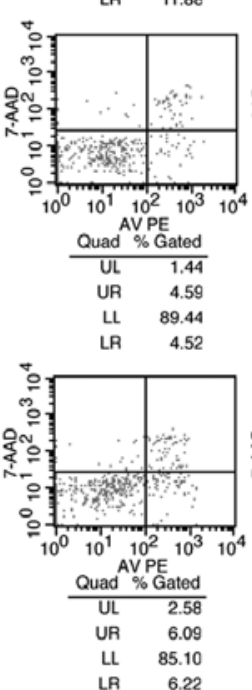

CFPAC-1/GR

B
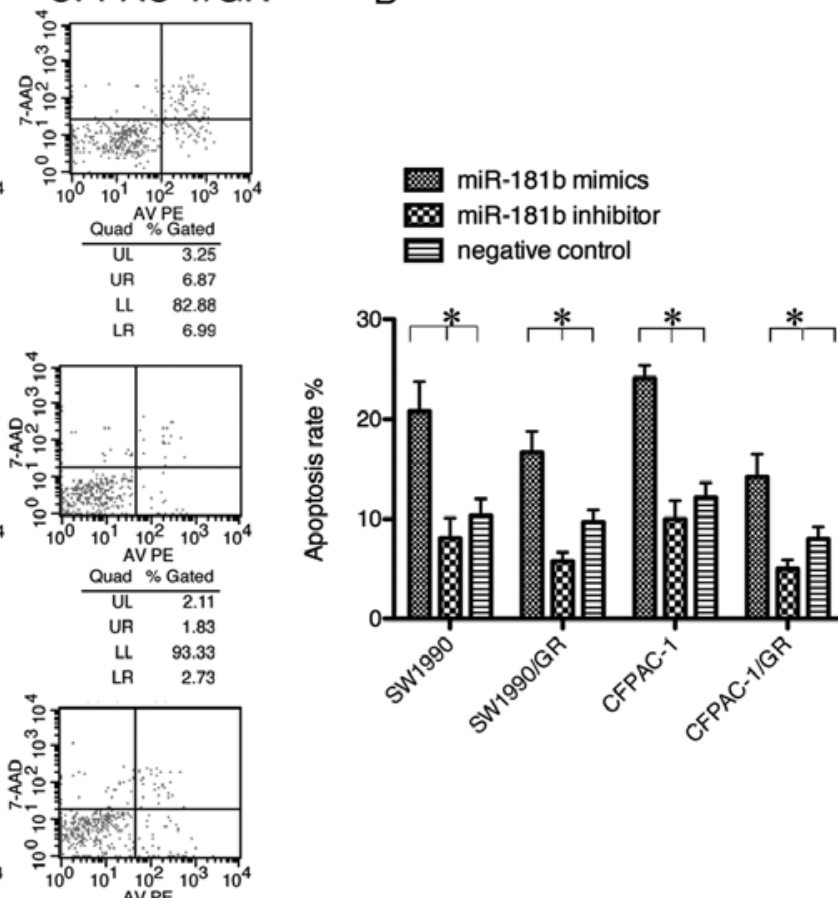

Figure 3. Effects of miR-181b on pancreatic ductal adenocarcinoma (PDAC) cell apoptosis. (A) Flow cytometric assay. Parental and drug-resistant cells transfected with miRNA-181b mimics manifested higher apoptotic rates, whereas miRNA 181b inhibitor-transfected cells showed significantly lower apoptotic rates than the control groups. miRNA-181b enhanced gemcitabine (0.1 $\mu \mathrm{M})$-induced apoptosis. Quad, quadrant; UL, upper left; UR, upper right; LL, lower left; LR, lower right. (B) Quantified data from (A). ${ }^{*} \mathrm{p}<0.05$.

fected with miRNA-181b mimics, inhibitor, or control, and then treated with a gemcitabine-conditioned medium $(1 \mu \mathrm{M})$ for $72 \mathrm{~h}$. The MTT assay showed that miRNA-181b mimics increased the gemcitabine sensitivity of these 4 cell lines (Table III and Fig. 2C).

miRNA-181b promotes the apoptosis of PDAC cells. To further elucidate gemcitabine sensitivity in PDAC cells, we performed a flow cytometric assay. Tumor cells were transiently transfected with miRNA-181b mimics, inhibitor or control, and then treated with gemcitabine $(0.1 \mu \mathrm{M})$. The data showed that miRNA-181b caused the PDAC cells to undergo apoptosis (Fig. 3).

miRNA-181b promotes gemcitabine sensitivity in PDAC cells in vivo. To confirm our in vitro data, we performed nude mouse xenograft assays. PDAC SW1990 and SW1990/GR cells were transfected with a lentivirus carrying miR-181b mimics or negative control sequences in vitro that were then transplanted into the pancreata of nude mice. Two weeks after tumor cell transplantation, gemcitabine was administered to the mice at $150 \mathrm{mg} / \mathrm{kg}$ twice weekly for 4 weeks. The tumor size in the mice in the miRNA-181b overexpression group was smaller than that in the mice of the null control group (Fig. 4).

miRNA-181b affects the gemcitabine resistance of PDAC cells by the downregulation of BCL-2 expression. To explore the underlying molecular events, we performed western blot analysis and found that miRNA-181b reduced the expression of BCL-2 protein in vitro and in tumor xenografts (Fig. 5B). We also performed ELISA to detect caspase-3 activity and found that caspase-3 activity increased with miRNA-181b overexpression, but was reduced following treatment with a miRNA-181b inhibitor (Fig. 5C). 


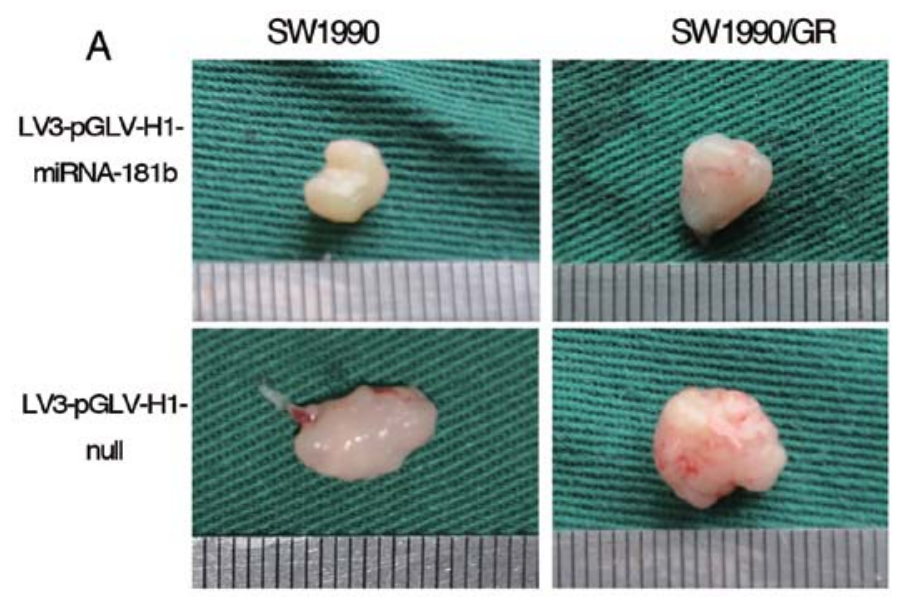

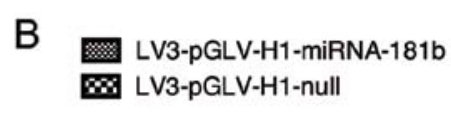

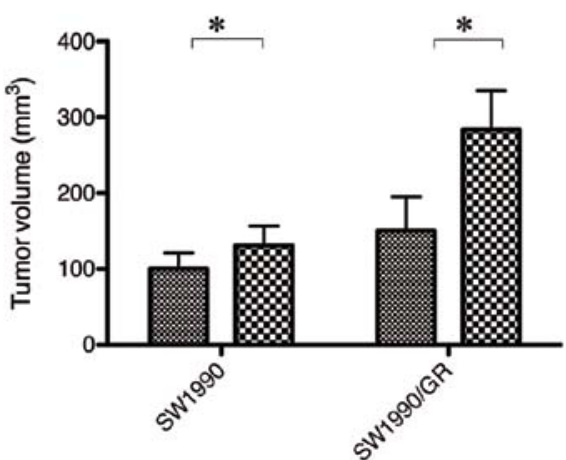

Figure 4. Effects of miR-181b on tumor xenograft growth. (A) Nude mouse xenograft assay. Pancreatic ductal adenocarcinoma (PDAC) cells were transfected with miR-181b lentivirus and then implanted into recipient mouse pancreata. Two weeks later, the mice were treated with $150 \mathrm{mg} / \mathrm{kg}$ gemcitabine twice a week for 4 weeks. Subsequently, tumor xenografts were removed and images were taken. The overexpression of miRNA-181b led to a significantly smaller tumor size in the normal and resistant cell lines. The images are representative of 6 specimens. (B) Tumor volume. ${ }^{*} \mathrm{p}<0.05$.

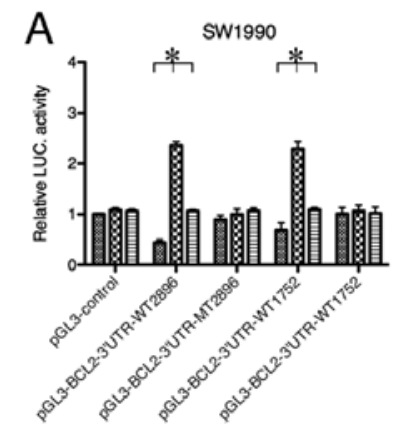

B

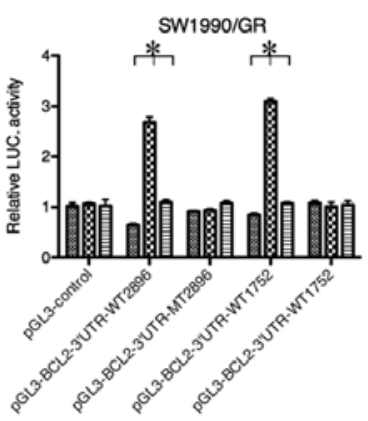

xenografts SW1990 SW1990/GR

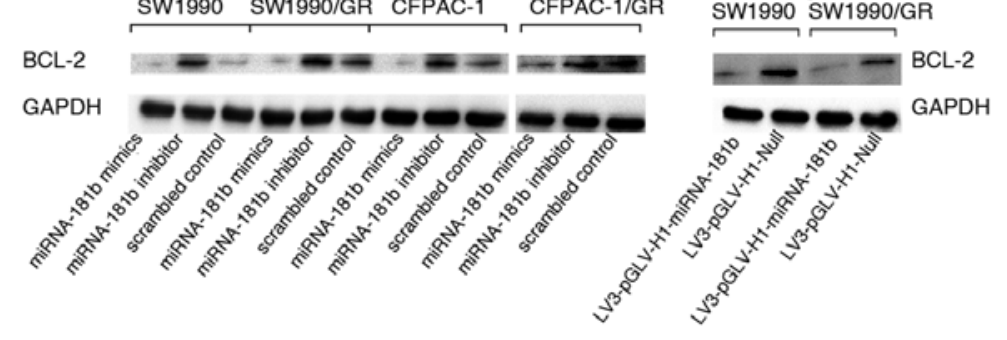

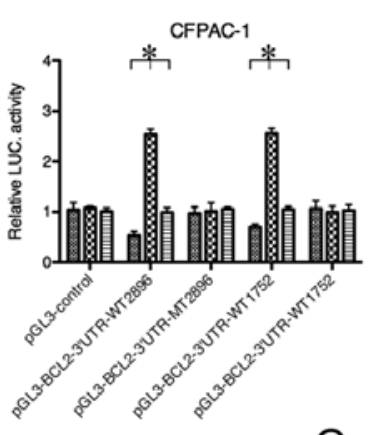

C
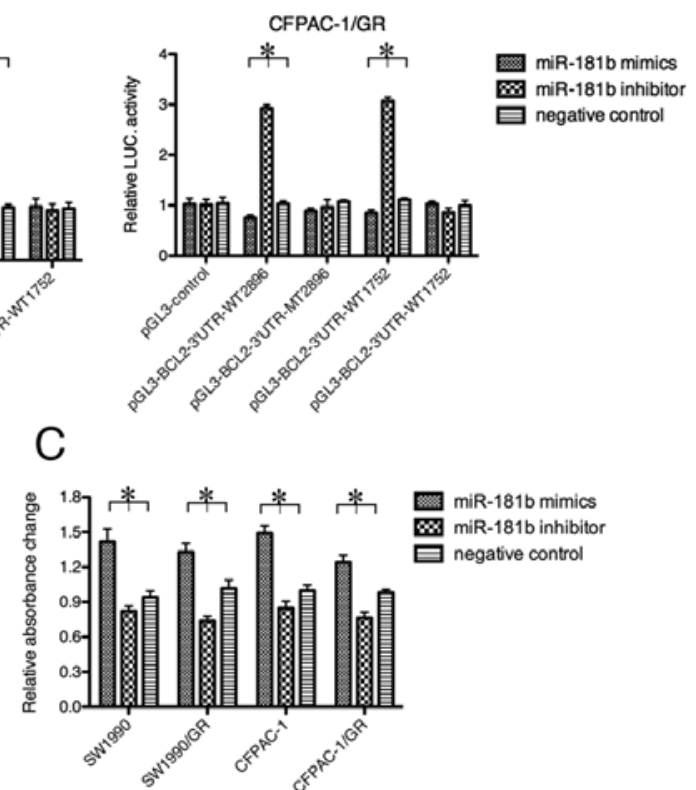

Figure 5. miR-181b targets BCL-2. (A) Dual-luciferase reporter assay. BCL-2 3'UTR sequences or mutated sequences were designed as MT2896, WT2896, MT1752 and WT1752, and then inserted into a pGL3 vector. Subsequently, they were co-transfected with miRNA-181b mimics, inhibitor, or a negative control into pancreatic ductal adenocarcinoma (PDAC) cells. The Renilla luciferase expression plasmid, pRL-TK, and the original pGL3 control were utilized as an internal and external standard, respectively. Luciferase activity was downregulated by miRNA-181b mimics, but elevated by a miRNA-181b inhibitor in the normal and resistant cell lines. Luciferase activity with the mutant sequences showed no significant changes among cells transfected with miRNA-181b mimics, inhibitor or negative control ( $\left.{ }^{*} \mathrm{p}<0.05\right)$. (B) Western blot analysis. PDAC cells were transiently transfected with miRNA-181b mimics, inhibitor, or control for $48 \mathrm{~h}$. Total cellular protein was extracted and blotted using a BCL-2 antibody. The data revealed that miRNA-181b overexpression reduced BCL-2 expression, whereas a miRNA-181b inhibitor increased BCL-2 levels. (C) ELISA. Caspase-3 activity was significantly increased by the exogenous expression of miRNA-181b and decreased by a miRNA-181b inhibitor in the parental and drug-resistant cells $\left({ }^{*} \mathrm{p}<0.05\right)$.

We also performed bioinformatics analyses and found there are 2 potential miR-181b target sites in the BCL-2 3'UTR. We performed a dual-luciferase reporter assay to assess whether BCL-2 is indeed a direct downstream target of miRNA-181b. The luciferase activity was significantly restrained by miRNA-181b mimics, while the miRNA-181b inhibitor led to an enhancement of luciferase activity in normal and resistant cell lines (Fig. 5A).

\section{Discussion}

In the present study, we investigated the effects of miRNA-181b on PDAC cell sensitivity to gemcitabine and the potential underlying molecular mechanisms. We found that although gemcitabine-resistant PDAC sublines expressed higher levels of miR-181b than the parental cell lines, miRNA-181b mimics significantly induced PDAC cell sensitivity to gemcitabine in 
both parental and drug-resistant PDAC cells in vitro and in nude mouse xenografts. By contrast, a miRNA-181b inhibitor led gemcitabine resistance in PDAC cells. miR-181b mimics also induced significantly higher levels of tumor cell apoptosis than the controls. Molecularly, miR-181b suppressed BCL-2 expression and induced caspase- 3 activity in PDAC cells Therefore, it can be concluded that miRNA-181b may be useful in the clinical treatment of gemcitabine-resistant PDAC.

In this study, we first established gemcitabine-resistant PDAC sublines and unexpectedly discovered that miR-181b expression was higher in drug-resistant cells when compared to parental cells, for an unknown reason. These data indicate that during the establishment of gemcitabine resistance, miR-181b itself is not sufficient to antagonize gemcitabine. By contrast, the transient transfection of a miR-181b mimics sensitizes PDAC cells to gemcitabine. This was further confirmed in parental cell lines, as well as in in vivo experiments using nude mice. In clinical practice, gemcitabine is frequently used as the first-line chemotherapy for PDAC. A previous study showed however, that gemcitabine had a response rate of $<20 \%$ (33). Thus, increasing drug sensitivity by miR-181b may be a novel tool for PDAC chemotherapy and improving patient survival. In this study, miR-181b increased the sensitivity of PDAC cells to gemcitabine and induced PDAC cell apoptosis. The evasion of apoptosis has long been acknowledged as one of the hallmarks of cancer (34). The induction of apoptosis could effectively control PDAC progression.

Recently, miRNA has been recognized to play a critical role in the regulation of cell apoptosis and chemosensitivity $(13,14)$. Functionally, miRNA can complementarily target the mRNAs of target genes, and thereby degrade or inhibit them from translating into proteins. Thus, the altered expression of miRNAs contributes to a variety of human diseases, such as inherited diseases, heart disease and cancer $(35,36)$. miRNA-181b has been reported to be involved in certain types of human malignancies. Tissue inhibitor of metalloprotease 3 (TIMP3) and BCL-2 mRNAs are major targets of miRNA-181b. Thus, the overexpression of miR-181b may lead to the downregulation of these genes and alter tumor progression and chemoresistance. Previous studies have shown that miRNA-181b suppresses TIMP3 expression in breast cancer and hepatocellular carcinoma cells, and facilitates tumor progression and chemoresistance $(28,30)$. By contrast, miRNA-181b expression has been shown to enhance the sensitivity of B-cell CLL, as well as that of gastric and lung cancer cells to chemotherapy through the downregulation of BCL-2 expression $(26,31)$. Moreover, in pancreatic cancer, miRNA-181b is considered a prospective biomarker for pre-operative diagnosis $(23,37,38)$. However, the mechanisms of miRNA-181b action in PDAC carcinogenesis and chemoresistance remain unclear.

A number of gene pathways, including the Akt, EGFR, SHH, Notch, MAPK and NFאB pathways, have been associated with chemoresistance (39). BCL-2 plays an important role in apoptosis and gemcitabine resistance $(13,40)$. BCL-2 protein maintains the integrity of the mitochondrial membrane by preventing caspase activation (e.g., caspase-3), resulting in cell survival (41). Transcriptionally, various kinases and transcription factors can induce BCL-2 expression $(13,42)$. As a target gene of miRNA-181b, BCL-2 facilitates cell survival against chemotherapy via the blockage of Bax/Bak-induced apoptosis $(18,26,31)$. A previous study showed that BCL-2 also participates in gemcitabine resistance in PDAC (43). Thus, our current data support the positive role of miR-181b in sensitizing PDAC cells to gemcitabine.

\section{References}

1. Siegel R, Ward E, Brawley O and Jemal A: Cancer statistics, 2011: the impact of eliminating socioeconomic and racial disparities on premature cancer deaths. CA Cancer J Clin 61: 212-236, 2011.

2. Li W, Ma Q, Liu J, et al: Hyperglycemia as a mechanism of pancreatic cancer metastasis. Front Biosci 17: 1761-1774, 2012.

3. Hanahan D and Weinberg RA: Hallmarks of cancer: the next generation. Cell 144: 646-674, 2011.

4. Garrido-Laguna I, Uson M, Rajeshkumar NV, et al: Tumor engraftment in nude mice and enrichment in stroma-related gene pathways predict poor survival and resistance to gemcitabine in patients with pancreatic cancer. Clin Cancer Res 17: 5793-5800, 2011.

5. Saiki Y, Yoshino Y, Fujimura H, et al: DCK is frequently inactivated in acquired gemcitabine-resistant human cancer cells. Biochem Biophys Res Commun 421: 98-104, 2012.

6. de Wolf C, Jansen R, Yamaguchi H, et al: Contribution of the drug transporter ABCG2 (breast cancer resistance protein) to resistance against anticancer nucleosides. Mol Cancer Ther 7: 3092-3102, 2008.

7. Schniewind B, Christgen M, Kurdow R, et al: Resistance of pancreatic cancer to gemcitabine treatment is dependent on mitochondria-mediated apoptosis. Int J Cancer 109: 182-188, 2004.

8. Kagawa S, Takano S, Yoshitomi H, et al: Akt/mTOR signaling pathway is crucial for gemcitabine resistance induced by Annexin II in pancreatic cancer cells. J Surg Res 178: 758-767, 2012.

9. Lu H, Buchan RJ and Cook SA: MicroRNA-223 regulates Glut4 expression and cardiomyocyte glucose metabolism. Cardiovasc Res 86: 410-420, 2010.

10. Ma F, Liu X, Li D, et al: MicroRNA-4661 upregulates IL-10 expression in TLR-triggered macrophages by antagonizing RNA-binding protein tristetraprolin-mediated IL-10 mRNA degradation. J Immunol 184: 6053-6059, 2010.

11. Ambros V: MicroRNA pathways in flies and worms: growth, death, fat, stress, and timing. Cell 113: 673-676, 2003.

12. Hummel R, Hussey DJ and Haier J: MicroRNAs: predictors and modifiers of chemo- and radiotherapy in different tumour types. Eur J Cancer 46: 298-311, 2010.

13. Lima RT, Busacca S, Almeida GM, Gaudino G, Fennell DA and Vasconcelos MH: MicroRNA regulation of core apoptosis pathways in cancer. Eur J Cancer 47: 163-174, 2011.

14. Schoof CR, Botelho EL, Izzotti A and Vasques Ldos R: MicroRNAs in cancer treatment and prognosis. Am J Cancer Res 2: 414-433, 2012.

15. Ratert N, Meyer HA, Jung M, et al: Reference miRNAs for miRNAome analysis of urothelial carcinomas. PLoS One 7: e39309, 2012.

16. Pallante P, Visone R, Ferracin M, et al: MicroRNA deregulation in human thyroid papillary carcinomas. Endocr Relat Cancer 13: 497-508, 2006.

17. Zanette DL, Rivadavia F, Molfetta GA, et al: miRNA expression profiles in chronic lymphocytic and acute lymphocytic leukemia. Braz J Med Biol Res 40: 1435-1440, 2007.

18. Visone R, Veronese A, Rassenti LZ, et al: miR-181b is a biomarker of disease progression in chronic lymphocytic leukemia. Blood 118: 3072-3079, 2011.

19. Xi Y, Formentini A, Chien M, et al: Prognostic values of microRNAs in colorectal cancer. Biomark Insights 2: 113-121, 2006.

20. Yan LX, Huang XF, Shao Q, et al: MicroRNA miR-21 overexpression in human breast cancer is associated with advanced clinical stage, lymph node metastasis and patient poor prognosis. RNA 14: 2348-2360, 2008.

21. Schaefer A, Jung M, Mollenkopf HJ, et al: Diagnostic and prognostic implications of microRNA profiling in prostate carcinoma. Int J Cancer 126: 1166-1176, 2010.

22. Xu X, Jia R, Zhou Y, et al: Microarray-based analysis: Identification of hypoxia-regulated microRNAs in retinoblastoma cells. Int J Oncol 38: 1385-1393, 2011.

23. Panarelli NC, Chen YT, Zhou XK, Kitabayashi N and Yantiss RK: MicroRNA expression aids the preoperative diagnosis of pancreatic ductal adenocarcinoma. Pancreas 41: 685-690, 2012. 
24. Ciafre SA, Galardi S, Mangiola A, et al: Extensive modulation of a set of microRNAs in primary glioblastoma. Biochem Biophys Res Commun 334: 1351-1358, 2005.

25. Chen L, Yang Q, Kong WQ, et al: MicroRNA-181b targets cAMP responsive element binding protein 1 in gastric adenocarcinomas. IUBMB Life 64: 628-635, 2012.

26. Dan Xia Z, Wei Z, Cheng F, et al: miR-181a/b significantly enhances drug sensitivity in chronic lymphocytic leukemia cells via targeting multiple anti-apoptosis genes. Carcinogenesis 33: 1294-1301, 2012.

27. Careccia S, Mainardi S, Pelosi A, et al: A restricted signature of miRNAs distinguishes APL blasts from normal promyelocytes. Oncogene 28: 4034-4040, 2009.

28. Wang B, Hsu SH, Majumder S, et al: TGFbeta-mediated upregulation of hepatic miR-181b promotes hepatocarcinogenesis by targeting TIMP3. Oncogene 29: 1787-1797, 2010.

29. Nakajima G, Hayashi K, Xi Y, et al: Non-coding MicroRNAs hsa-let-7g and hsa-miR-181b are associated with chemoresponse to $\mathrm{S}-1$ in colon cancer. Cancer Genomics Proteomics 3: 317-324, 2006.

30. Lu Y, Roy S, Nuovo G, et al: Anti-microRNA-222 (anti-miR-222) and $-181 \mathrm{~B}$ suppress growth of tamoxifen-resistant xenografts in mouse by targeting TIMP3 protein and modulating mitogenic signal. J Biol Chem 286: 42292-42302, 2011.

31. Zhu W, Shan X, Wang T, Shu Y and Liu P: miR-181b modulates multidrug resistance by targeting BCL2 in human cancer cell lines. Int J Cancer 127: 2520-2529, 2010.

32. An Y, Yao J, Wei JS, et al: Establish a gemcitabine-resistant pancreatic cancer cell line SW1990/GZ and research the relationship between SW1990/GZ and pancreatic cancer stem cell Zhonghua Wai Ke Za Zhi 48: 999-1003, 2010 (In Chinese).

33. Friess H, Langrehr JM, Oettle $\mathrm{H}$, et al: A randomized multicenter phase II trial of the angiogenesis inhibitor Cilengitide (EMD 121974) and gemcitabine compared with gemcitabine alone in advanced unresectable pancreatic cancer. BMC Cancer 6: 285, 2006.
34. Hanahan D and Weinberg RA: The hallmarks of cancer. Cell 100: 57-70, 2000.

35. Osman A: MicroRNAs in health and disease - basic science and clinical applications. Clin Lab 58: 393-402, 2012.

36. Fillat $C$ and Altafaj X: Gene therapy for Down syndrome. Prog Brain Res 197: 237-247, 2012.

37. Ren Y, Gao J, Liu JQ, et al: Differential signature of fecal microRNAs in patients with pancreatic cancer. Mol Med Rep 6: 201-209, 2012.

38. Liu J, Gao J, Du Y, et al: Combination of plasma microRNAs with serum CA19-9 for early detection of pancreatic cancer. Int $J$ Cancer 131: 683-691, 2012.

39. Hung SW, Mody HR and Govindarajan R: Overcoming nucleoside analog chemoresistance of pancreatic cancer: a therapeutic challenge. Cancer Lett 320: 138-149, 2012.

40. Cory S and Adams JM: The Bcl2 family: regulators of the cellular life-or-death switch. Nat Rev Cancer 2: 647-656, 2002.

41. De Botton S, Sabri S, Daugas E, et al: Platelet formation is the consequence of caspase activation within megakaryocytes. Blood 100: 1310-1317, 2002.

42. Zhao Y, Shen S, Guo J, et al: Mitogen-activated protein kinases and chemoresistance in pancreatic cancer cells. J Surg Res 136: 325-335, 2006.

43. Dong J, Zhao YP, Zhou L, Zhang TP and Chen G: Bcl-2 upregulation induced by miR-21 via a direct interaction is associated with apoptosis and chemoresistance in MIA $\mathrm{PaCa}-2$ pancreatic cancer cells. Arch Med Res 42: 8-14, 2011. 